\title{
Numerical parametric study of three-dimensional tunnel response due to overlying circular basement excavation
}

\author{
SHI, Jiangwei ${ }^{1, \text { a }}$ \\ ${ }^{1}$ Key Laboratory of Geomechanics and Embankment Engineering of the Ministry of Education, \\ Hohai University, 1 Xikang Road, Nanjing 210098, China.
}

aceshijiangwei@163.com

\begin{abstract}
Keywords: Numerical parametric study; three-dimensional; tunnel response; circular basement Abstract: Deep basement excavation inevitably induces stress changes in the ground leading to soil movements, which may cause adverse effects on existing tunnels. Although basement-tunnel interaction has attracted increasing attention recently, the influence of circular basement excavation on metro tunnels is rarely investigated. A systematic numerical parametric study is conducted to investigate the influence of excavation diameter and cover-to-tunnel diameter on three-dimensional deformation mechanisms of underlying tunnels. The tunnel heave and transverse tensile strain at basement centerline reach maximum values when the excavation diameter is seven times of the final excavation depth. If a basement excavation with a small diameter is assumed to be a two-dimensional problem, tunnel heave and transverse tensile strain are grossly overestimated.
\end{abstract}

\section{Introduction}

Underground metro systems such as tunnels are constructed to relieve traffic jams. For public convenience, increasing numbers of basements are being excavated to the side of tunnels or above them. The safety and serviceability of tunnels may be affected by adjacent basement excavation. Burford $^{[1]}$ reported that the maximum tunnel heave due to basement excavation in London was up to $60 \mathrm{~mm}$. Moreover, Cracks were observed in the reinforced concrete segments of the shield tunnel ${ }^{[2]}$. Obviously, basement excavation can induce significant adverse effects on existing tunnels.

By conducting field monitoring ${ }^{[1-2]}$, centrifuge tests ${ }^{[3]}$ and numerical analyses ${ }^{[4]}$, many studies have been conducted to investigate ground movement and tunnel responses due to nearby rectangular basement excavation. It is not uncommon to encounter triangular ${ }^{[5]}$ and circular ${ }^{[6]}$ basements. Tan ${ }^{[6]}$ investigated ground movements due to a large-scale circular basement excavation in Shanghai. The excavation diameter and excavation depth were $130 \mathrm{~m}$ and $34 \mathrm{~m}$, respectively. Compared with rectangular and triangular basements, arching effects in circular basements result in smaller lateral wall movements. Thus, response of existing tunnel located underneath circular basement is controlled by vertical stress relief rather than inward wall movements. However, investigation of tunnel response due to circular excavation was limited. In this study, a numerical parametric study was conducted to investigate the effects of circular basement excavation on an existing tunnel. Soil response was described by an advanced constitutive model which can capture soil small-strain stiffness.

\section{Three dimensional modeling}

\section{Numerical analysis program}

The final excavation depth and thickness of retaining wall were designed as $9 \mathrm{~m}$ and $0.96 \mathrm{~m}$, respectively. The diameter and wall thickness of an existing tunnel were $6.2 \mathrm{~m}$ and $0.35 \mathrm{~m}$ which were common values for metro tunnels. To simulate a medium-dense soil, the relative sand density was selected as $68 \%$. To investigate three-dimension tunnel responses, the excavation diameter varied from $18 \mathrm{~m}$ to $73 \mathrm{~m}$ (i.e., $2-7$ times of the final excavation depth). The cover-to-tunnel diameter ratio varied from 2.0 to 3.0. In total, 18 numerical runs were performed to evaluate three-dimensional tunnel responses due to basement excavation. 


\section{Finite element mesh and boundary conditions}

Fig.1 shows the finite element mesh of circular basement-tunnel interaction. The finite element software ABAQUS was adopted. Solid elements were used to simulate the sand stratum and the retaining wall, while shell elements were used to model the existing tunnel. Roller and pin supports were applied at the four vertical sides and the base of the mesh, respectively. Perfect contacts were assumed at the soil-structure interfaces. To eliminate boundary effects, the clearest distance between the retaining wall and the boundary of the mesh was larger than twice of the final excavation depth.
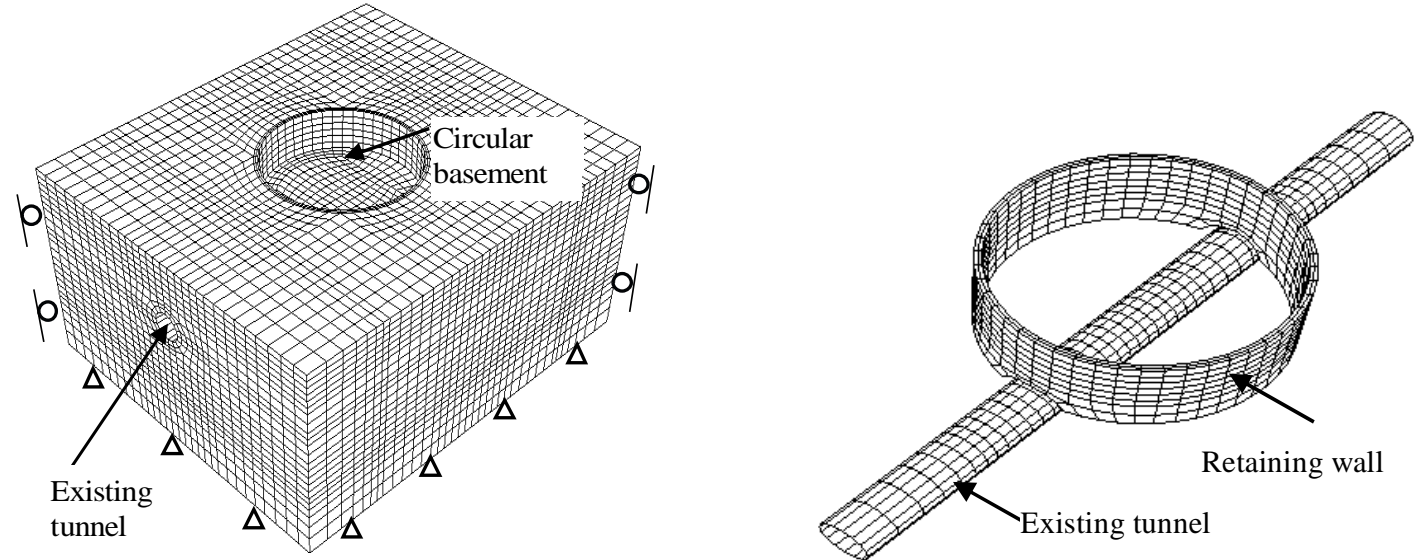

Fig. 1 Finite element mesh and boundary conditions

\section{Constitutive models and model parameters}

The soil behaviour was described by a user-defined hypoplastic sand (HP) model incorporated into the software ABAQUS. The governing equation of the soil model is defined by Eq. (1):

$$
\stackrel{\circ}{\mathrm{T}}=f_{\mathrm{s}}\left(\boldsymbol{L}: \boldsymbol{D}+f_{\mathrm{d}} \boldsymbol{N}\|\boldsymbol{D}\|\right)
$$

where $\boldsymbol{L}$ is a fourth-order tensor, $\boldsymbol{N}$ is a second-order tensor, $\boldsymbol{D}$ is rate of deformation, factors $f_{\mathrm{s}}$ and $f_{\mathrm{d}}$ capture dependency of soil response on mean stress level and relative density, respectively. The hypoplastic sand model proposed by Von Wolffersdorff ${ }^{[7]}$ was adopted in this study. It requires eight material parameters $\left(\varphi_{c}^{\prime}, h_{s}, n, e_{d 0}, e_{c 0}, e_{i 0}, \alpha\right.$ and $\left.\beta\right)$. Niemunis \& Herle ${ }^{[8]}$ incorporated the intergranular strain concept into the hypoplastic sand model to capture the effects of strain and stress path dependency on soil stiffness. Another five material parameters $\left(m_{T}, m_{R}, R, \beta_{r}\right.$ and $\left.\kappa\right)$ are required for the modification. Parameters of $\varphi_{c}^{\prime}, h_{s}, n, e_{d 0}, e_{c 0}, e_{i 0}, \alpha$ and $\beta$ are taken as $30^{\circ}, 2.6 \mathrm{GPa}, 0.27,0.61,0.98$, $1.10,0.14$ and 3, respectively. Moreover, parameters of $m_{T}, m_{R}, R, \beta_{r}$ and $\left.\kappa\right)$ were taken as $8,4,2 \times 10^{-5}$, 0.1 and 1.0 , respectively.

\section{Validation of soil model and model parameters}

Fig. 2 compares the measured and computed stress-strain curves of Toyoura sand at two relative sand densities. The soil parameters of $\varphi_{c}^{\prime}, h_{s}, n, e_{d 0}, e_{c 0}$ and $e_{i 0}$ were directly taken from Herle and Gudehus $^{[9]}$. Parameters of $\alpha$ and $\beta$ were calibrated by triaxial test results ${ }^{[10]}$. By using the soil parameters $\left(\varphi^{\prime}{ }_{c}, h_{s}, n, e_{d 0}, e_{c 0}, e_{i 0}, \alpha\right.$ and $\left.\beta\right)$ summarized in previous section, the HP model can capture soil strain softening and state dependent dilatancy behavior. Agreements between the measured and computed results demonstrated that the adopted constitutive model parameters were reasonable.

Fig. 3 compares the measured and computed small-strain stiffness degradation curves of Toyoura sand. After fixing the eight soil parameters for the basic model, remaining five parameters were calibrated by small-strain stiffness curve. Using the five parameters summarized in previous section, the computed stiffness degradation curve generally agreed well with the measured one when soil strain was larger than $10^{-3} \%$. In the triaxial tests, local transducers were installed to measure soil strain. However, local transducer could only give a reliable measurement of soil strain ranging from $10^{-3} \%$ to $1 \%$. Thus, soil strain less than $10^{-3} \%$ might not be properly captured by the local transducers. 


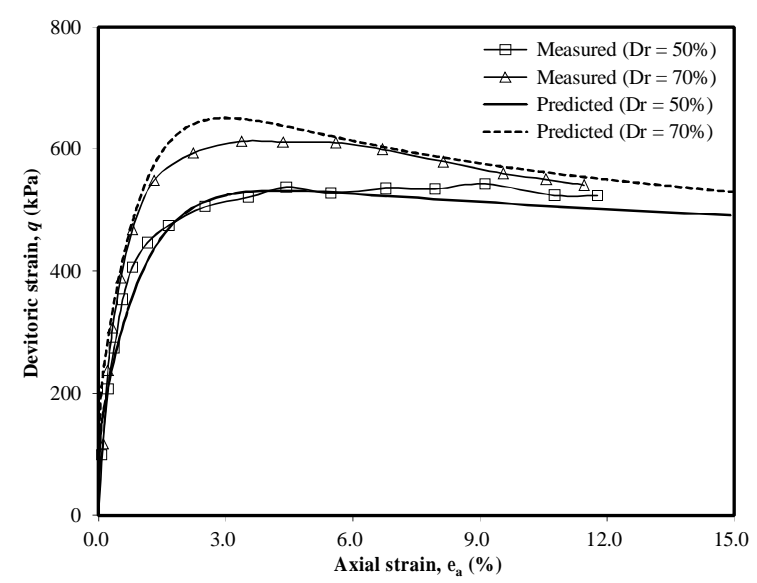

Fig.2 Comparison of stress-strain curves

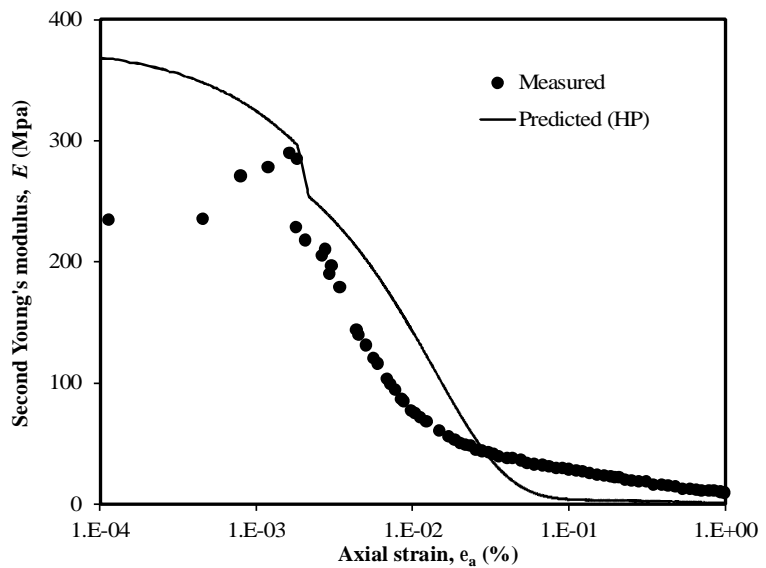

Fig.3 Comparison of small-strain stiffness curves

\section{Influence of excavation diameter on tunnel heave and transverse tensile strain}

Fig.4 shows variations of normalized tunnel heave with excavation geometry. At a given C/D ratio, the maximum tunnel heave increases with an increase in the excavation diameter, but at a reduced rate. When the excavation diameter is larger than four times of the final excavation depth, the maximum tunnel heave exceeds the allowable movement limit (i.e., $10 \mathrm{~mm}$ ). Due to arching effects in the circular basements, the underlying tunnel response is induced by vertical stress relief only. As an increase in the excavation diameter, constraints from three dimensional effects on tunnel response are reduced. Thus, the maximum tunnel heave at the basement centerline varies from three dimensional response to two dimensional response. When the normalized excavation diameter $\left(D_{B} / H_{e}\right)$ varies from 6 to 7 , the increase in the maximum tunnel heave is less than $1 \%$. It is indicated that the tunnel heave at basement center reaches a maximum value when the value of $D_{B} / H_{e}$ is equal to 7 . If the basement-tunnel interaction at the center of a small excavation (i.e., $D_{B} / H_{e}=2.0$ ) is assumed as the plane strain problem (i.e., $D_{B} / H_{e}=7.0$ ), the heave induced in the tunnel is overestimated by $100 \%$. Thus, if three-dimensional effects of basement excavation are not considered, a grossly estimation of tunnel response is obtained. At a given normalized excavation diameter $\left(D_{B} / H_{e}\right)$, excavation-induced maximum tunnel heave decreases as an increase in the cover-to-tunnel diameter ratio. This is because stiffness of soil surrounding a deep tunnel is large, and vertical stress relief in deep soil is small.

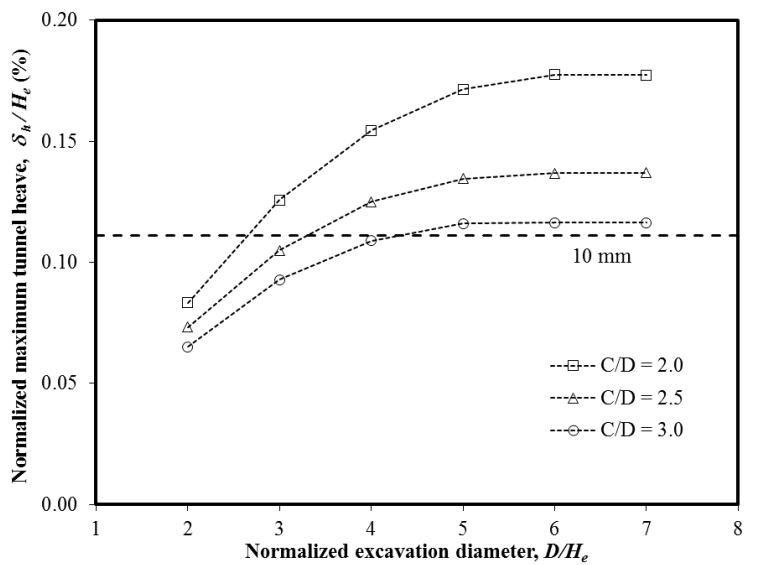

Fig.4 Maximum tunnel heave

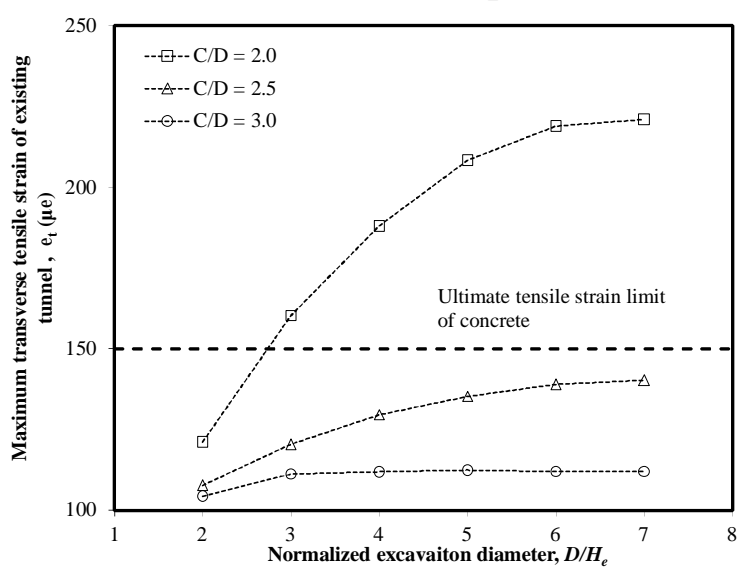

Fig.5 Maximum transverse tensile strain of tunnel

Fig. 5 shows variations of maximum transverse tensile strain of tunnel with excavation geometry. At a given $C / D$ ratio, the maximum transverse tensile strain of tunnel increases with an increase in the excavation diameter, but at a reduced rate. As an increase in the $\mathrm{C} / \mathrm{D}$ ratio, the maximum tensile strain of tunnel decreases rapidly. This is consistent with the observation as shown in Fig. 4. At a given C/D ratio, the increase in the maximum transverse tensile strain of tunnel is less than $1 \%$ when the $D_{B} / H_{e}$ varies from 6 to 7 . Thus, the maximum transverse tensile strain of tunnel at basement center is close to a plane strain problem when the $D_{B} / H_{e}$ is 7 . If the basement-tunnel interaction at the center of a small 
excavation (i.e., $D_{B} / H_{e}=2.0$ ) is assumed as the plane strain problem (i.e., $D_{B} / H_{e}=7.0$ ), the maximum transverse tensile strain induced in the tunnel is overestimated by $80 \%$. This demonstrates again the importance of consideration of three-dimensional effects of basement excavation. For shallow tunnels $(C / D=2.0)$, the maximum tensile strain of tunnel exceeds the allowable limit of concrete when the excavation diameter is larger than twice of the final excavation depth. Thus, countermeasures may be adopted to alleviate basement excavation effects when the excavation geometry is large.

\section{Conclusions}

A numerical parametric study is conducted to investigate the influence of circular basement excavation on three-dimensional tunnel deformation mechanisms. The maximum heave and transverse tensile strain of tunnel decreases rapidly as an increase in the cover-to-tunnel diameter ratio. The tunnel heave and transverse tensile strain at basement centerline reach maximum values when the excavation diameter reaches seven times of the final excavation depth. If the basement-tunnel interaction at the center of a small excavation is assumed as a plane strain problem, the heave and transverse tensile strain of existing tunnels are overestimated by $100 \%$ and $80 \%$, respectively. When the excavation diameter is larger than four times of the final excavation depth, the maximum tunnel heave exceeds the allowable movement limit (i.e., $10 \mathrm{~mm}$ ). Thus, countermeasures may be adopted to alleviate basement excavation effects when the excavation geometry is large.

\section{References}

[1] D. Burford. Heave of tunnels beneath the Shell Centre, London, 1959-1986. Géotechnique, 38 (1): 135-137 (1988).

[2] C.T. Chang, C.W. Sun, S.W. Duann, R.N. Hwang. Response of a Taipei Rapid Transit System (TRTS) tunnel to adjacent excavation. Tunn Undergr Space Technol., 16 (3): 151-158 (2001).

[3] C.W.W. Ng, J.W. Shi, Y. Hong. Three-dimensional centrifuge modelling of basement excavation effects on an existing tunnel in dry sand. Can Geotech J, 50(8): 874-888 (2003).

[4] Huang, X., Schweiger, H.F., Huang, H.W. Influence of deep excavations on nearby existing tunnels. Int J Geomech, 13 (2): 170-180 (2013).

[5] J.W. Shi, G.B. Liu, P. Huang. Interaction between a large triangular excavation and adjacent structures in Shanghai soft clay. Tunn Undergr Space Technol, 50: 282-295 (2015).

[6] Y. Tan, D Wang. Characteristics of a large-scale deep foundation pit excavated by the central-island technique in Shanghai soft clay.I: bottom-up construction of the central cylindrical shaft. J. Geotech. Geoenviron. Eng., 139(11): 1875-1893 (2013).

[7] P.A. Von Wolffersdorff. A hypoplastic relationship for granular material with a predefined limit state surface, Mech Cohes-Frict Mater, 1, pp. 251-271 (1996).

[8] A. Niemunis, I. Herle. Hypoplastic model for cohensionless soils with elastic strain range. Mech Cohes-Frict Mater, 2, pp. 279-299 (1997).

[9] I. Herle, G. Gudehus. Determination of parameters of a hypoplastic constitutive model from properties of grain assemblies. Mech Cohes-Frict Mater, 4 (5): 461-486 (1999).

[10] K. Maeda, K. Miura. Relative density of dependency of mechanical properties of sands. Soils Found, 39 (1): 69-79 (1999). 